T lymphocytes, followed by DNA synthesis and cell division. After division clones of cells stimulated by antigen become small again, recirculate, and can have a rapid secondary response on re-exposure to the antigen.

Exposure to antigen also causes $T$ lymphocytes to liberate a series of products that affect the functions of other cells. Included in the products are a macrophage chemotactic factor, a macrophage migration inhibitory factor, a macrophage aggregation factor, lymphotoxin (which kills certain other cells), and interferon. These products can reproduce many of the features found in delayed hypersensitivity lesions, including macrophage infiltration. As a result of cell-mediated immune reactions macrophages show increased capacity to kill intracellular organisms such as Listeria monocytogenes.

A second lineage of lymphocytes is now recognized and known as B lymphocytes. They have immunoglobulin (antibody) on their outer membranes, and respond to antigen by transforming into antibody-secreting cells. Most antigens, such as foreign proteins, stimulate B lymphocytes only inefficiently by direct contact. A second signal produced by interaction of nearby $T$ lymphocytes with antigen is required for stimulation of B lymphocytes to proliferate and transform into antibody-secreting cells. Thus most antigens are thymus-dependent. But some naturally occurring antigens, such as pneumococcus polysaccharide or bacterial endotoxin, stimulate B lymphocytes as well in the absence as in the presence of $T$ lymphocytes; they are therefore known as thymus-independent antigens.

The functions of $T$ lymphocytes can thus be defined as participation in various types of cell-mediated immune reactions and helping in the formation of antibody. The importance of $T$ lymphocytes is evident from the severity of the syndrome of selective thymus deficiency. Because this defect allows the retention of grafted cells, attempts have been made to restore immune function by intramuscular inoculation of foreign fetal thymus cells. ${ }^{34}$ Cells from fetuses 13 to 16 weeks old have been used to reduce the danger of graft-versus-host reactions, which can be fatal after inoculation of thymus cells from stillborn babies or older persons. The children with grafted thymuses have had their $T$ lymphocyte activities restored and been clinically improved.

Some degree of immunological reconstitution of thymectomized mice can be achieved by inserting into them thymus cells enclosed in diffusion chambers with pores too small to permit emigration of cells but large enough to allow release of secretory products. R. W. Steele and his colleagues $^{5}$ have enclosed 13-week-old fetal thymus in a diffusion chamber in a child with thymic aplasia. The child's lymphocytes became responsive to phytohaemagglutinin, but she died 9 days after the graft. Though these results are compatible with the interpretation that a humoral thymus factor can bring about maturation of $\mathbf{T}$ lymphocytes in man, other effects cannot be excluded. In any case, injection of thymus cells is much more likely to restore immune function than enclosure of cells in a diffusion chamber, and it is to be hoped that, as these cases are recognized and treated, further experience of injecting fetal thymus cells will be gained.

1 Miller, J. F. A. P., Lancet, 1961, 2, 748.

2 diGeorge, A. M., Fournal of Pediatrics, 1965, 67, 907.

${ }^{3}$ Cleveland, W. W., Fogel, B. J., Brown, W. T., and Kay, H. E. M.,

' August, C. S., Levey, R. H., Berkel, A. I., Rosen, F. S., and Kay, H. E. M., Lancet, 1970, 1,1080 .

${ }^{5}$ Steele, R. W., et al., New England fournal of Medicine, 1972, 287, 787.

\section{Care of the Dying}

Even if there is some truth in the assertion that death has replaced sex as the taboo subject in our society at least doctors still recognize that it is one of their major concerns. Indeed, the last few years have seen growing medical interest in the case of the dying: the creation of hospices for terminal care; better understanding of the special psychological needs and problems at this time; and the realization that such care also involves continuing support of the bereaved. Nevertheless, clearly there is still much room for improving these services and on 29 November 1972 the Department of Health held a nationwide symposium on the subject. At page 29 of this week's B.M.F. we print shortened versions of the papers read at the symposium, which will be published in full by the D.H.S.S. together with the discussion.

Three main themes emerged from these contributions. Firstly, despite recent developments and the fact that many patients still die peacefully at home, we need many more specialist terminal-care units-possibly, according to one speaker, 12 beds for every half million people. Secondly, we need to continue to examine alternative ways of providing accommodation for the dying. In some areas the best solution is to provide long-term units attached to special or general hospitals or totally on their own. In other areas short-term units may admit the patient for further treatment, to relieve the strain on the family temporarily, or in the final stages. In yet others a few beds in a community hospital enable death to be placed in its proper perspective of the cycle of life from which many townsmen have become all too isolated.

The final main theme of the conference was the importance of team work in terminal care. Today the concept of the team seems to be a cliché brought out all too often in discussing any medical problem. But in the care of the dying undoubtedly a team led by the family doctor which includes district nurses, members of the social services and voluntary organizations, and the clergy has a valuable supportive role; and it should be easier to assemble teams like this after the forthcoming reorganization of the health services. Perhaps in no other field of medical treatment are minor details so important as in terminal care, and doctors will find many of the points raised at the admirable D.H.S.S. conference of great value.

\section{Perinatal Therapeutics and Toxicology}

Any substance in solution in the blood of a pregnant woman can pass into the blood of the embryo or fetus. The question is not whether but how fast and by what mechanism any particular substance crosses the placental "barrier"l and, with increasing emphasis, how its effects on the offspring compare with its effects on the adult. The thalidomide disaster made it only too clear that its effects on the embryo, at least, may be very different. And it has long been known that analgesic and anaesthetic agents administered to the mother during labour not only cross her blood-brain barrier but also tend to cross the placental barrier, ${ }^{2}$ and so to depress the nervous system of the fetus, with potentially disastrous effects 
on its ability to establish satisfactory respiration after delivery. Potentially toxic substances may also be transmitted to the baby in breast milk. ${ }^{3}$

More recently two new types of toxic effects on the fetus have been described. Aspirin and promethazine have been shown to interfere with platelet aggregation in the blood of the baby to a much greater extent than in that of the mother across whose placenta they have been transmitted. ${ }^{4}$ That barbiturate withdrawal symptoms in the baby have been described only this year 67 is presumably due to the fact that their onset may be delayed for up to 14 days after birth and so go unnoticed, whereas the similar effect in babies of narcotic addicts is usually apparent within 24 hours-and it was recognized 13 years ago.8

Clearly a combination of laboratory studies and thorough clinical observation must be employed in any attempt to define the possible toxic effects on the fetus of any drug which is intended to have a therapeutic effect on its mother. A report by R. D. G. Milner and S. K. Chouksey ${ }^{9}$ of the effects of fetal exposure to diazoxide is an excellent example of such an investigation of a new form of treatment. Diazoxide in a dose of $200-400 \mathrm{mg}$ per day was administered to four women with severe pre-eclamptic toxaemia for between 19 and 69 days before delivery. It appeared to have a beneficial effect in helping to prolong each of the pregnancies to a stage at which the baby had an improved chance of survival. The diazoxide, which was present in the cord plasma at about half the maternal level, was excreted in the baby's urine during the first week of life. There was no adverse effect on the blood pressure or blood sugar level during the first day or two of life, nor on blood counts, immunoglobulins, or later ocular development. There was some retardation of ossification in the wrist of one baby, but all four showed some degree of alopecia, and one developed hypertrichosis lanuginosa (a reported complication of diazoxide treatment of hypoglycaemia in childhood). These abnormalities of hair growth were first noted at the age of 1 week and were still present at the time of the report, when the babies were aged 5-12 months, so that their prognosis was uncertain. But they do not appear to have been sufficiently severe to outweigh the apparent advantages of the treatment. It seems likely that in the future there will be an increasing demand for similar careful balancing of perinatal therapeutic and toxic effects of drugs.

1 Page, E. W., American Fournal of Obstetrics and Gynecology, 1957, 74, 705. 2 Moya, F., and Thorndike, V., American fournal of Obstetrics and Gynecology, 1962, 84, 1778.

3 Smithells, R W. and Morgan, D. M., Practitioner, 1970, 204, 14

Bleyer, W. A., and Breckenridge, R. T., fournal of American Medical Association, 1970, 213, 2049.

5 Corby, D. G., and Schulman, I., fournal of Pediatrics, 1971, 79, 307.

6 Desmond, M. M., Schwanecke, R. P., Wilson, G. S., Yasunaga, S., and Burgdorff, I., Fournal of Pediatrics, 1972, 80, 190.

7 British Medical fournal, 1972, 4, 63.

${ }^{8}$ Cobrinik, R. W., Hood, R. T., and Chusid, E., Pediatrics, 1959, 24, 288.

Milner, R. D. G., and Chouksey, S. K., Archives of Disease in Childhood, $1972,47,537$.

\section{Cutaneous Polyarteritis}

Skin lesions of various types may be found in polyarteritis nodosa, an incidence of $25 \%$ often being cited. 1 They are frequently non-specific both clinically and histologically, the diagnosis being established only by signs of the disease in other organs. However, the skin changes can at times be striking and characteristic. Recently P. Borrie ${ }^{2}$ has reviewed
102 cases of polyarteritis nodosa from one hospital. Seventeen of them had cutaneous lesions, of which 12 showed recurrent subcutaneous nodules, often with patchy but widespread mottling of the skin usually called livedo reticularis or livedo racemosa. The nodules are usually tender, sometimes extremely painful, and may go on to ulceration or gangrene.

It should be emphasized that the nodules are the lesions which show the characteristic histology of polyarteritis. Livedo reticularis by itself is by no means even suggestive of polyarteritis nodosa. It is due to dilatation of small vessels and follows their network pattern in the skin, and it may have many causes, including especially disease of the underlying medium-sized arteries. ${ }^{3}$ Erythema ab igne, which follows the same pattern, is caused quite differently, by external heat. Patchy livedo reticularis, implying patchy arterial disease, is often said to be strongly suggestive of polyarteritis nodosa, but it has many other causes, including arterial emboli, thrombocythaemia (an important cause which may present in this way, and has a reasonable prognosis only if adequately treated), and various types of arteritis. The commonest type of livedo is best called idiopathic, and its relationship, if any, to polyarteritis nodosa is unproved. In this type of livedo without nodules biopsy specimens generally fail to show a histology suggestive of polyarteritis. Such patients may have crippling ulceration 45 and may also have widespread arterial disease presenting as multiple cerebral thromboses, coronary artery disease, and in other ways. 6

One important finding in Borrie's ${ }^{2}$ series is that the large majority of his patients showing livedo reticularis and nodules and a histology of polyarteritis had no evidence of disease in the lungs or kidneys, though lesions in muscles and nerves with fever and a raised erythrocyte sedimentation rate were rather common. The long-term prognosis for life and even for eventual regression of the cutaneous lesions was good. As with most types of arteritis, the aetiology of this condition is obscure, but there did seem to be more than a chance association with streptococcal infections in some of his cases, and also particularly in childhood. ${ }^{7}$

Borrie makes a good case for the separation of this group with cutaneous disease as a distinct clinical entity and considers that cases in which the skin lesions have the histological appearance of polyarteritis nodosa bear a good prognosis. Its relationship to the other commoner types of cutaneous vasculitis ${ }^{8}$ shows similar uncertainties as that between discoid and systemic lupus erythematosus or morphoea and systemic sclerosis.

These patients usually need corticosteroids, but, since the prognosis is good, heroic dosage with these or other drugs to achieve complete control is not indicated. The vogue for treating various types of cutaneous vasculitis with dapsone has not yet been assessed in these patients. The role of anticoagulants or low-molecular-weight dextran infusions, 9 which may be more successful in those cases of livedo reticularis without nodules, is also at present unknown.

1 Ketron, L. W., and Bernstein, J. C., Archives of Dermatology and Syphilo$\log y, 1939,40,929$.

2 Borrie, P., British fournal of Dermatology, 1972, 87, 87.

3 Champion, R. H., British fournal of Dermatology, 1965, 77, 167.

4 Feldaker, M., Hines, E. A., and Kierland, R. R., Archives of Dermatology, 1955, 72, 31 .

${ }^{5}$ Feldaker, M., Hines, E. A., and Kierland, R. R., Circulation, 1956, 13, 196.

- Sneddon, I. B., British Fournal of Dermatology, 1965, 77, 180.

7 Bradford, W. D., Cook, C. D., Vawter, G. F., Fournal of Paediatrics,

1962, 60, 266.
8 Borrie, P., and Stansfield, A., in Modern Trends in Dermatology, 3, p. 167, ed. R. M. B. MacKenna. London, Butterworth, 1966.

- Issroff, S. W., and Whiting, D. A., British fournal of Dermatology, 1971, 85, Supplement 7, 26. 